\title{
EXPERIMENTAL VALIDATION OF A SELF-ADJUSTING ACTIVE COMPLIANCE CONTROLLER FOR MULTIPLE ROBOTS HANDLING AN OBJECT
}

\author{
Christian von Albrichsfeld ${ }^{1}$, and Henning Tolle
}

\author{
Darmstadt University of Technology, Institute of Control Engineering, \\ Department of Control Systems Theory \& Robotics \\ Landgraf-Georg-Strasse 4, D-64283 Darmstadt \\ E-Mail: tolle@rt.e-technik.tu-darmstadt.de
}

\begin{abstract}
This paper presents the experimental validation of a self-adjusting active compliance controller for $n$ robots handling a concerning its compliant behaviour partly unknown flexible object. The control strategy is based on the decomposition of the $6 n$-dimensional position/force space and includes a feedforward and feedback level. For adjusting the controller to the in general unknown flexible behaviour, which is the main problem of the controller design, a quasi-static model of the system is derived for different contact cases of the object and a procedure is presented, which by use of this model is capable of determining the compliance of the considered system and therefore of adjusting the controller. Experiments with two pumatype robots show the applicability of the self-adjusting control strategy. Copyright $\odot 1998$ IFAC
\end{abstract}

Keywords: self-tuning control, force-position control, admittance control, compliance control, decoupling problems, multi-arm robots, intelligent control, robot control

\section{INTRODUCTION}

By use of multiple robot manipulators for handling an object the performance of the task execution can be improved, especially when the object might be very bulky, heavy, or flexible. In space robotics cases like this can be found at tasks like the capturing of a satellite for maintainance or repair purposes or the building of a space platform, which requires the assembly of many long beams. A number of control schemes have been proposed by the robotics community for the case of two or more robots firmly grasping and handling an object. It has been shown that control methods like hybrid position/force control (Hayati, 1986; Uchiyama and Dauchez, 1988), active stiffness/compliance control (Bruhm and Neusser, 1987), or model-based dynamic control (Tarn, 1986; Svinin and Uchiyama, 1994) achieve better results than pure position control or master/slave control approaches. But for obtaining a good performance a detailed knowledge of the complex static and for the model based dynamic controller also dynamic model of the robots and the object is needed. In general the handling of concerning its parameters partly unknown objects can not be done by these approaches.

\footnotetext{
1 now with ITT Automotive Europe GmbH, Guerickestr. 7, 60488
} Frankfurt, Germany. Email: Christian.Albrichsfeld@auto.ittind.com
Therefore recently simplified decentralized structures based on impedance (Szewczyk et al., 1996) or compliance control (Perdereau et al., 1996), which need only rough models of the system, and learning or adaptive control schemes (Pandian et al., 1994; von Albrichsfeld et al., 1995) have been introduced.

This paper extends an active compliance control scheme presented in (von Albrichsfeld, 1996), which is capable of adjusting its parameters to the unknown system compliance, by discussing different contact cases of the object, namely a contact, limiting the object's motion in all directions (clamped object), in some directions, and as well an unconstrained object. The applicability of the self-adjusting controller is verified by experiments with two 6-dof robots for the last case, the free object.

\section{MATHEMATICAL MODEL OF THE SYSTEM}

The system under consideration - an object grasped firmly by multiple robot manipulators - is shown in figure $1^{2}$. The small translational and rotational displace-

\footnotetext{
2 It should be denoted, that the derived model and also the presented self-adjusting control scheme can be used for the general case of $n$ robots handling an object. For sake of clear arrangement all pictures show only the simple case of two robots grasping an object.
} 
ments of the object at the grasping frames and the external contact frame created by the forces being applied at the respective points are represented as generalized 6 dimensional springs ${ }^{3}$. The robots as well can be mod-

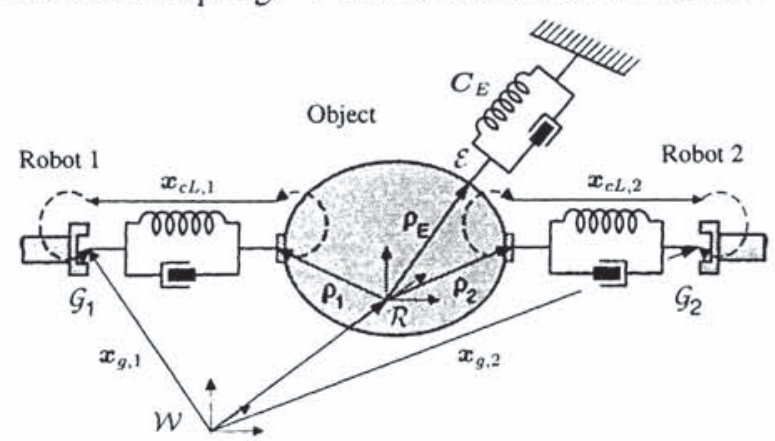

Figure 1. Virtual object with concentrated compliance.

eled in the same manner by concentrating their distributed compliance at the end effectors. They are combined with the compliance of the object, leading to an entire spring extension of $\boldsymbol{x}_{c, i}=\boldsymbol{x}_{c L, i}+\boldsymbol{x}_{c R, i}$, where $\boldsymbol{x}_{c R, i}$ denotes the elastic extension of the respective robot. The considered model of the whole system can be found in figure 1 by replacing the elastic object displacements $x_{c L, i}$ by the entire elastic displacements $x_{c, i}$ and the grasp frames $\mathcal{G}_{i}$ including the respective vectors $\boldsymbol{x}_{g, i}$ by the end effector frames of the rigid part of the robots $\mathcal{H}_{0 i}$ including the respective vectors $\boldsymbol{x}_{\mathcal{H} 0, i}$. As can be derived from the above mentioned equations of the system with distributed parameters the static displacements of the introduced springs are solely a function of the forces applied by the robots.

For deriving the stiffness matrix $S \in \Re^{6 n \times 6 n}$ of the whole system, denoting the relationship between the small combined displacement of the robots $\delta x_{\mathcal{H} 0}=$ $\left(\delta \boldsymbol{x}_{\mathcal{H} 0,1}^{\mathrm{T}}, \delta \boldsymbol{x}_{\mathcal{H} 0,2}^{\mathrm{T}}, \ldots, \delta \boldsymbol{x}_{\mathcal{H} 0, n}^{\mathrm{T}}\right)^{\mathrm{T}} \in \Re^{6 n}$ and force differences $\delta \boldsymbol{f}_{\mathcal{H}}=\left(\delta \boldsymbol{f}_{\mathcal{H}, 1}^{\mathrm{T}}, \delta \boldsymbol{f}_{\mathcal{H}, 2}^{\mathrm{T}}, \ldots, \delta \boldsymbol{f}_{\mathcal{H}, n}^{\mathrm{T}}\right)^{\mathrm{T}} \in \Re^{6 n}$ with $\delta f_{\mathcal{H}}=-S \cdot \delta x_{\mathcal{H} 0}$, the vector of combined arm displacement $\delta \boldsymbol{x}_{\mathcal{H} 0}$ is decomposed. The first partitioning scheme is based upon the rigid Jacobian matrix $\boldsymbol{J}_{g}=$ $\underset{i=1}{n}\left(\boldsymbol{J}_{g_{\mathrm{v}}}\right)=\left[\boldsymbol{J}_{g_{1}}^{\mathrm{T}} \boldsymbol{J}_{g_{2}}^{\mathrm{T}} \ldots \boldsymbol{J}_{g_{n}}^{\mathrm{T}}\right]^{\mathrm{T}} \in \Re^{6 n \times 6}$ of the object. It is composed of the Jacobians of each grip frame

$$
\text { with } \quad \boldsymbol{J}_{g_{i}}=\left[\begin{array}{cc}
\boldsymbol{I}_{3 \times 3} & \boldsymbol{\Omega}^{\mathrm{T}}\left(\boldsymbol{\rho}_{i}-\boldsymbol{\rho}_{E}\right) \\
\boldsymbol{O}_{3 \times 3} & \boldsymbol{I}_{3 \times 3}
\end{array}\right] \text {, }
$$

where $O \in \Re^{3 \times 3}$ is the null matrix and $\Omega(\cdot) \in \Re^{3 \times 3}$ is an operator such that $\Omega(\rho) \cdot b \equiv \rho \times b$. The Jacobian spans the space of rigid body motion $\mathrm{R}_{e}=\mathcal{R}\left(\boldsymbol{J}_{g}\right) \in$ $\Re^{6}$, i.e. the space of external motions and forces, whereas its nullspace defines the space of elastic deformations $\mathrm{R}_{i}=\mathcal{N}\left(\boldsymbol{J}_{g}^{\mathrm{T}}\right) \in \Re^{6(n-1)}$, i.e. the space of internal motions and forces (see figure 3 ).

With these introduced spaces we can derive the stiffness matrix $\boldsymbol{S}$ of the whole system: The combined arm displacement can be expressed as sum of the rigidly

\footnotetext{
3 In general the object has to be modeled with distributed parameters (Svinin and von Albrichsfeld, 1995), but due to the fact that we are only interested in the behaviour at certain points of the object we use
} a simplified static model with concentrated compliance. transformed displacement of the object's contact frame $\delta \boldsymbol{x}_{E}$ and the combined spring displacement $\delta \boldsymbol{x}_{c}=$ $\left(\delta \boldsymbol{x}_{c, 1}^{\mathrm{T}}, \delta \boldsymbol{x}_{c, 2}^{\mathrm{T}}, \ldots, \delta \boldsymbol{x}_{c, n}^{\mathrm{T}}\right)^{\mathrm{T}} \in \Re^{6 n}$, which is given by Hooke's law $\delta x_{c}=-C_{L R} \cdot \delta f_{\mathcal{H}}$ as a function of the reaction forces acting on the robot end effectors, where $C_{L R} \in \Re^{6 n \times 6 n}$ is the combined compliance matrix for the object and the robots in the case of the object being fixed at the external contact point $\mathcal{E}$, i.e. $\delta \boldsymbol{x}_{E} \equiv \boldsymbol{O}$ :

$$
\delta \boldsymbol{x}_{\mathcal{H} 0}=\boldsymbol{J}_{g} \cdot \delta \boldsymbol{x}_{E}-\boldsymbol{C}_{L R} \cdot \delta \boldsymbol{f}_{\mathcal{H}}
$$

The motion of the external contact point has to be expressed in accordance to the contact case of the object. Figure 2 shows the system. Let us consider, that the

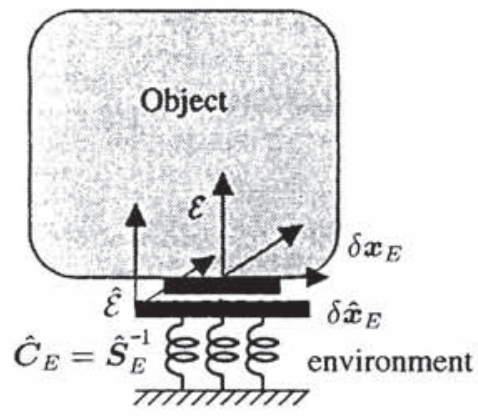

Figure 2. External contact of the object.

object is constrained by the contact in $m$ out of the 6 possible directions of motion. With $B_{x} \in \Re^{6 \times(6-m)}$ being the base of the remaining degrees of freedom of the object motion the relative displacements between the two coordinate frames $\mathcal{E}$ and $\hat{\mathcal{E}}$ can be parametrized as $\delta \boldsymbol{x}_{E}-\delta \hat{\boldsymbol{x}}_{E}=\boldsymbol{B}_{x} \delta \boldsymbol{x}_{e}$, where $\delta \boldsymbol{x}_{e} \in \Re^{6-m}$ are the components of the relative motion with respect to the base $\boldsymbol{B}_{x}$. According to Mason, forces can only be applied in a space orthogonal to the motion space. With $B_{f} \in \Re^{6 \times m}$ being the base of this orthogonal space $\left(\mathcal{R}\left(\boldsymbol{B}_{f}\right)=\right.$ $\mathcal{N}\left(\boldsymbol{B}_{x}^{\mathrm{T}}\right)$ ), the contact forces can be parametrized as follows: $\delta \boldsymbol{f}_{E}=\boldsymbol{B}_{f} \delta \boldsymbol{f}_{e}$, where $\delta \boldsymbol{f}_{e} \in \Re^{m}$ are the components of the forces with respect to the base $B_{f}$. By use of this parametrization the displacement $\delta \hat{x}_{E}$ follows as $\delta \hat{\boldsymbol{x}}_{E}=-\hat{\boldsymbol{C}}_{E} \cdot \delta \boldsymbol{f}_{E}=-\hat{\boldsymbol{C}}_{E} \cdot \boldsymbol{B}_{f} \delta \boldsymbol{f}_{e}$ with $\hat{\boldsymbol{C}}_{E} \in \Re^{6 \times 6}$ being the nonsingular compliance matrix of the environment at the external contact point. The displacement of the external contact point $\delta \boldsymbol{x}_{E}$ can then be written as

$$
\delta \boldsymbol{x}_{E}=\boldsymbol{B}_{x} \delta \boldsymbol{x}_{e}-\hat{\boldsymbol{C}}_{E} \cdot \boldsymbol{B}_{f} \delta \boldsymbol{f}_{e} .
$$

This equation describes a decomposition of the motion/force space of the external contact. By inserting $\delta x_{E}$ from (3) into (2) the combined arm displacement follows as

$$
\delta \boldsymbol{x}_{\mathcal{H} 0}=\boldsymbol{J}_{g} \boldsymbol{B}_{x} \delta \boldsymbol{x}_{e}-\boldsymbol{J}_{g} \hat{C}_{E} \delta \boldsymbol{f}_{E}-C_{L R} \delta \boldsymbol{f}_{\mathcal{H}},
$$

which by use of the force balance at the external contact point $\left(\delta \boldsymbol{f}_{E}-\boldsymbol{J}_{g}^{\mathrm{T}} \cdot \delta \boldsymbol{f}_{\mathcal{H}}=\mathbf{0}\right)$ leads to

$$
\begin{aligned}
\delta \boldsymbol{x}_{\mathcal{H} 0} & =\boldsymbol{J}_{g} \boldsymbol{B}_{x} \delta \boldsymbol{x}_{e}-\left(\boldsymbol{J}_{g} \hat{\boldsymbol{C}}_{E} \boldsymbol{J}_{g}^{\mathrm{T}}+\boldsymbol{C}_{L R}\right) \delta \boldsymbol{f}_{\mathcal{H}} \\
& =\boldsymbol{J}_{g} \boldsymbol{B}_{x} \delta \boldsymbol{x}_{e}-\left(\boldsymbol{J}_{g} \hat{\boldsymbol{C}}_{E} \boldsymbol{J}_{g}^{\mathrm{T}}+\boldsymbol{C}_{L R}\right) \boldsymbol{B}_{\text {eif }} \delta \boldsymbol{f}_{\text {eif }}
\end{aligned}
$$

The last equation is found by using the parametrization $\delta \boldsymbol{f}_{\mathcal{H}}=\boldsymbol{B}_{\text {eif }} \delta \boldsymbol{f}_{\text {eif }}$ of the arm forces. This follows from the previous mentioned two decompositions of the motion/force spaces. By transformation of the 
motion/force space of the external contact point to the arm quantities $\delta x_{\mathcal{H} 0}$ and $\delta \boldsymbol{f}_{\mathcal{H}}$ a decomposition of the external motion/force space into a position controlled and a force controlled subspace is conducted (see figure 3). According to (6) the position controlled external

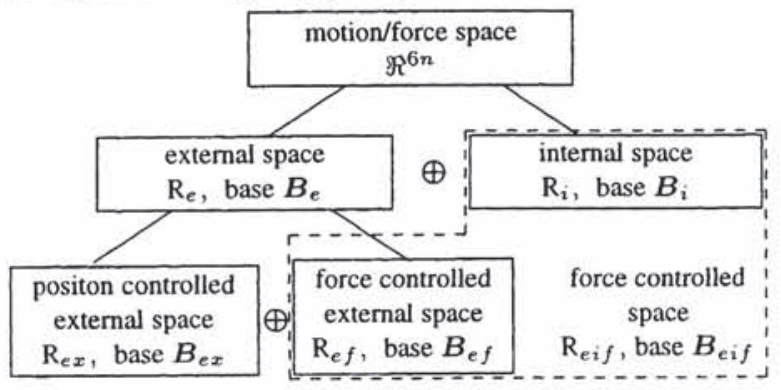

Figure 3. Decomposition of the motion/force space of the combined arm quantities into subspaces.

space is spanned by the matrix product $\boldsymbol{J}_{g} \boldsymbol{B}_{x}\left(\mathrm{R}_{e x}=\right.$ $\mathcal{R}\left(\boldsymbol{J}_{g} \boldsymbol{B}_{x}\right)$, with the base $\left.\boldsymbol{B}_{e x}\right)$ and the force controlled external space is defined by the part of the nullspace of $\mathrm{R}_{e x}$ in the external space $\mathrm{R}_{e f}=\mathcal{R}\left(\boldsymbol{J}_{g}\left(\boldsymbol{J}_{g}^{\mathrm{T}} \boldsymbol{J}_{g}\right)^{-1} \boldsymbol{B}_{f}\right)$.

The direct sum of the internal space $\mathrm{R}_{i}$, where forces can be applied in every direction, and the force controlled external space $\mathrm{R}_{\text {ef }}$ forms the space of all admissible forces, the whole force controlled space, being the nullspace of the position controlled external space $\mathrm{R}_{\text {eif }}=\mathcal{N}\left(\boldsymbol{B}_{x}^{\mathrm{T}} \boldsymbol{J}_{g}^{\mathrm{T}}\right)$ with it's base $\boldsymbol{B}_{\text {eif }}=\left[\boldsymbol{B}_{i} \boldsymbol{B}_{e f}\right] \epsilon$ $\Re^{6 \times(6 n \times 6(n-1)+m)}$.

By exploiting the orthogonality of the position and force controlled subspaces $\left(\mathrm{R}_{e x} \perp \mathrm{R}_{\text {eif }} \Leftrightarrow\left(\boldsymbol{J}_{g} \boldsymbol{B}_{x}\right)^{\mathrm{T}} \cdot \boldsymbol{B}_{\text {eif }}=\right.$ $\left.\boldsymbol{O}_{(6-m) \times(6(n-1)+m)}\right)$ the stiffness matrix of the whole system can be derived from (6).

$\boldsymbol{S}=\boldsymbol{B}_{\text {eif }}\left(\boldsymbol{B}_{\text {eif }}^{\mathrm{T}}\left(\boldsymbol{J}_{g} \hat{C}_{E} \boldsymbol{J}_{g}^{\mathrm{T}}+\boldsymbol{C}_{L R}\right) \boldsymbol{B}_{\text {eif }}\right)^{-1} \boldsymbol{B}_{\text {eif }}^{\mathrm{T}}$ (7)

As can be seen, the stiffness matrix defines a projection onto the space of admissible forces $\mathrm{R}_{\text {eif }}=\mathcal{R}\left(\boldsymbol{B}_{i}\right) \oplus$ $\mathcal{R}\left(\boldsymbol{B}_{\text {ef }}\right)$, shown in figure 4 by the outermost pair of arrows, leading from the left oval, representing the arm

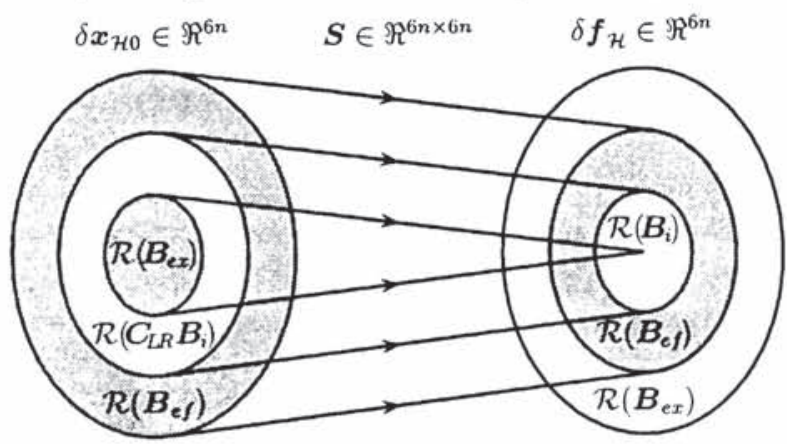

Figure 4. Singular stiffness mapping.

displacement to the right one, representing the arm forces. Equation (7) also shows, that an arm displacement in the motion controlled external space $\mathrm{R}_{e x}$ is mapped onto the nullspace $\left(\boldsymbol{S} \boldsymbol{J}_{g} \boldsymbol{B}_{x} \delta \boldsymbol{x}_{e}=\mathbf{0}\right)$, displayed by the innermost pair of arrows. The third projection shown in the figure is derived from another representation of the stiffness matrix not shown in this paper (see (von Albrichsfeld, 1998)).
Because all arm displacements are mapped by the stiffness matrix onto the space of admissible forces, spanned by $\boldsymbol{B}_{\text {eif }}$, the stiffness matrix has a rank defizit of (6$m$ ), which means it is singular apart from the case of a fixed object, where $m=6$. Therefore it's inverse, the compliance matrix, which is needed in the control strategy, can only be defined as a generalized inverse $S^{\#}$, defining the minimum $M_{x}$-norm, $M_{f}$-least-squares solution of the system $\left(-\Delta f_{\mathcal{H}}=S \Delta x_{\mathcal{H}}\right)$,

$$
\begin{aligned}
S^{\#}= & M_{x}^{-1} B_{e i f}\left(B_{e i f}^{\mathrm{T}} M_{x}^{-1} B_{e i f}\right)^{-1} B_{e i f}^{\mathrm{T}} \\
& \cdot\left(\boldsymbol{J}_{g} \hat{C}_{E} \boldsymbol{J}_{g}^{\mathrm{T}}+C_{L R}\right) \\
& \cdot \boldsymbol{B}_{e i f}\left(\boldsymbol{B}_{e i f}^{\mathrm{T}} \boldsymbol{M}_{f} \boldsymbol{B}_{e i f}\right)^{-1} B_{e i f}^{\mathrm{T}} \boldsymbol{M}_{f}
\end{aligned}
$$

and in particular the Moore-Penrose Pseudoinverse (with $M_{x}=M_{f}=I_{6 n \times 6 n}$ ) is given by

$$
\boldsymbol{S}^{+}=\boldsymbol{B}_{e i f} \boldsymbol{B}_{e i f}^{\mathrm{T}}\left(\boldsymbol{J}_{g} \hat{\boldsymbol{C}}_{E} \boldsymbol{J}_{g}^{\mathrm{T}}+\boldsymbol{C}_{L R}\right) \boldsymbol{B}_{e i f} \boldsymbol{B}_{e i f}^{\mathrm{T}} \text {. }
$$

So the inverse system can be written as

$$
\delta \boldsymbol{x}_{\mathcal{H} 0}=-\boldsymbol{S}^{\#} \delta \boldsymbol{f}_{\mathcal{H}} .
$$

\section{COORDINATION STRATEGY}

For controlling the two-arm system, a coordination strategy, presented in (von Albrichsfeld, 1996), is used, (see Figure 5). The strategy is suited for motion servoed industrial robots equipped with wrist force/torque sensors for measuring the applied arm forces.

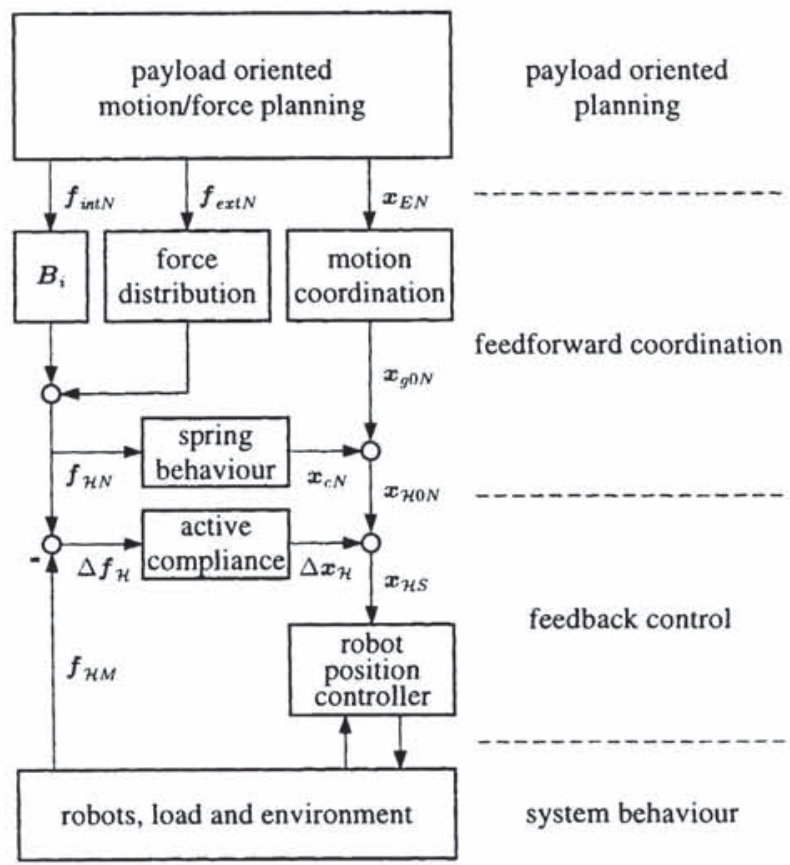

Figure 5. Coordination strategie.

The planning module provides the nominal payload motion $\boldsymbol{x}_{E N}(t) \in \Re^{6}$, the desired internal forces $\boldsymbol{f}_{\text {int } N}(t) \in$ $\Re^{6(n-1)}$, and by use of the dynamic model of the object, the nominal external forces to be applied to the object $\boldsymbol{f}_{\text {ext } N}(t)=\boldsymbol{f}_{E N}(t)+\boldsymbol{f}_{\text {Acc } N}(t)+\boldsymbol{f}_{\text {Grav } N}(t) \in \Re^{6}$, taking into account external forces to be excerted to the environment, acceleration and gravitational forces as a function of time $t$. 
The feed-forward coordinator is divided into a motion coordinator and a force/torque coordinator. The first one derives a combined nominal motion trajectory $\boldsymbol{x}_{g 0 N}(t)$, where $\boldsymbol{x}_{g 0 N}=\left(\boldsymbol{x}_{g 0 N, 1}^{\mathrm{T}}, \boldsymbol{x}_{g 0 N, 2}^{\mathrm{T}}, \ldots, \boldsymbol{x}_{g 0 N, n}^{\mathrm{T}}\right)^{\mathrm{T}} \in \Re^{6 n}$ represents the generalized coordinates of the grip frames, by a "rigid" transformation from the object external contact frame to the grapple frames corresponding to the vectors $\rho_{i}-\rho_{E}(\mathrm{i}=1 . \mathrm{n})$. Feedforward force/torque coordination is achieved by a linear force distribution law $\boldsymbol{f}_{\mathcal{H} N}=\boldsymbol{J}_{g}^{\mathrm{T} \#} \cdot \boldsymbol{f}_{\text {ext } N}+\boldsymbol{B}_{i} \cdot \boldsymbol{f}_{\text {int } N}$. External forces/torques are distributed by the matrix $\boldsymbol{J}_{g}^{\mathrm{T} \#}$, the $\{1,2,3\}$-generalized inverse (see (Doty et al., 1993)) of the transposed Jacobian $\boldsymbol{J}^{\mathrm{T}}$ matrix, satisfying the condi-

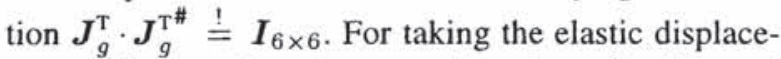
ments of the springs into consideration a feedforward loop is incorporated, calculating the spring displacements $\boldsymbol{x}_{c N}$ as function of the arm forces to be applied ${ }^{4}$.

The feedback loop choosen as active compliance law, calculates corrections of the nominal motion trajectory $\Delta \boldsymbol{x}_{\mathcal{H}}$ out of tracking errors of the nominal force $\Delta \boldsymbol{f}_{\mathcal{H}}$. It is made up of two controllers, one for controlling internal and one for external forces, each constructed as a mixture of generalized spring and damper terms

$$
\begin{aligned}
\Delta \boldsymbol{x}_{\mathcal{H}} & =\Delta \boldsymbol{x}_{\mathcal{H} \text { int }}+\Delta \boldsymbol{x}_{\mathcal{H} \text { ext }} \\
& =-\left[k_{\text {int }}\left(1+\frac{1}{T_{\text {int }} \cdot s}\right) \boldsymbol{S}^{\sharp} \boldsymbol{P}_{\text {int }}\right. \\
& \left.+k_{\text {ext }}\left(1+\frac{1}{T_{\text {ext }} \cdot s}\right) \boldsymbol{S}^{\#} \boldsymbol{P}_{\text {ext }}\right] \Delta \boldsymbol{f}_{\mathcal{H}},
\end{aligned}
$$

where $k_{i n t}, T_{i n t}, k_{\text {ext }}$ and $T_{\text {ext }}$ are scalar gain coefficients, each pair forming a PI-controller. The generalized Inverse $\boldsymbol{S}^{\#}$ is used as a static filter for decoupling the forces created by the nondiagonal parameters of the system stiffness matrix $S$ and achieving unit gain in the force controlled diagonal directions. $\boldsymbol{P}_{\text {int }}$ and $\boldsymbol{P}_{\text {ext }}$ are projection matrices, which confine the activity of the compliance controller to the respective internal and external force controlled space respectively while maintaining unit gain in the open loop matrix (see (von Albrichsfeld, 1998) for details).

In conventional stiffness or compliance control schemes the stiffness matrices and their inverses, the compliance matrices of the system, in detail, the robots, sensors, and the object, are supposed to be known. But in practice especially when handling unknown objects, they are only known in a very rough approximation. Due to couplings between displacements acting in one direction and forces/torques arising in other directions, the system compliance can not be determined easily.

Therefore the goal of a selfadjusting controller is to accomodate the control law to the unknown system compliance during execution of a task by observing the mea-

\footnotetext{
4 It should be denoted, that in contrast to the last section, where quasistatical behaviour was assumed for derivation of the stiffness matrix, the forces $f_{\mathcal{H} N}$ contain due to gravitational and accelerational forces, calculated by use of the dynamic model of the object and distributed to the arms by the force distribution law, also an external part, and therefore are not in the space of $\mathrm{R}_{e i f}$.
}

sured forces/torques and positions of the robots. Due to the fact, that no external devices should be used to measure the position of the object's reference point, and due to the singularity of the stiffness matrix $S$, the system is underdetermined for calculating the individual compliance matrices of the object and the robots $C_{L R}$ and the compliance matrix of the external contact point $\hat{C}_{E}$, or to measure the used generalized inverse $S^{\#}$ directly.

But as we want to control forces only in the space of admissible forces, it is not necessary to determine the compliance behaviour of the individual springs, but only the mapping onto the space of admissible forces $R_{e i f}$. By a simple transformation of the observed variables the underdetermined $6 n$-dimensional problem space can be transformed to an unambiguous $6(n-1)+m$-dimensional subspace

$$
\begin{aligned}
S_{e i f}^{-1} & \equiv B_{e i f}^{\mathrm{T}}\left(\boldsymbol{J}_{g} \hat{C}_{E} \boldsymbol{J}_{g}^{\mathrm{T}}+\boldsymbol{C}_{L R}\right) \boldsymbol{B}_{e i f} \\
& =\boldsymbol{B}_{\text {eif }}^{\mathrm{T}} \boldsymbol{S}^{\#} \boldsymbol{B}_{e i f}=\boldsymbol{B}_{\text {eif }}^{\mathrm{T}} \boldsymbol{S}^{+} \boldsymbol{B}_{\text {eif }} ; \\
\boldsymbol{S}_{\text {eif }}^{-1} & \in \Re^{(6(n-1)+m) \times(6(n-1)+m)} .
\end{aligned}
$$

This transformation is done by multiplying the measured variables $\delta x_{\mathcal{H} 0}$ and $\delta f_{\mathcal{H}}$ in (10) with $\boldsymbol{B}_{\text {eif }}^{\mathrm{T}}$, making use of the properties of the orthonormal base matrix,

$$
\begin{gathered}
\qquad \delta f_{e i f}=B_{e i f}^{\mathrm{T}} \delta \boldsymbol{f}_{\mathcal{H}} ; \quad \delta \boldsymbol{x}_{e i f}=\boldsymbol{B}_{e i f}^{\mathrm{T}} \delta \boldsymbol{x}_{\mathcal{H} 0},(16) \\
\text { leading to } \quad \delta \boldsymbol{x}_{e i f}=-\boldsymbol{S}_{\text {eif }}^{-1} \delta \boldsymbol{f}_{\text {eif }} .
\end{gathered}
$$

In terms of this transformed compliance matrix $S_{e i f}^{-1}$ the generalized inverse used in the controller (12) can be written as follows

$$
\begin{aligned}
\boldsymbol{S}^{\#}= & \boldsymbol{M}_{x}^{-1} \boldsymbol{B}_{e i f}\left(\boldsymbol{B}_{\text {eif }}^{\mathrm{T}} \boldsymbol{M}_{x}^{-1} \boldsymbol{B}_{e i f}\right)^{-1} \cdot \boldsymbol{S}_{e i f}^{-1} \\
& \cdot\left(\boldsymbol{B}_{\text {eif }}^{\mathrm{T}} \boldsymbol{M}_{f} \boldsymbol{B}_{\text {eif }}\right)^{-1} \boldsymbol{B}_{\text {eif }}^{\mathrm{T}} \boldsymbol{M}_{f},
\end{aligned}
$$

and in particular the Moore-Penrose Pseudoinverse (with $M_{x}=M_{f}=I$ ) is given by

$$
S^{+}=B_{e i f} S_{e i f}^{-1} B_{e i f}^{\mathrm{T}} .
$$

Due to the fact, that the forces are controlled only in the space of the admissible forces, the generalized inverse is also used in the block called spring behaviour instead of the actual spring displacements in the feedforward level of the coordination strategy (see figure 5).

$$
\boldsymbol{x}_{c N}=-\boldsymbol{S}^{\#} \boldsymbol{f}_{\mathcal{H} N} .
$$

Also here the generalized inverse is expressed in terms of the transformed compliance matrix.

\section{EXPERIMENTAL RESULTS}

For showing the applicability of the introduced control approach in combination with the scheme for determining the generalized inverse of the system compliance matrix experiments have been conducted with two puma-type robots carrying a load. The experimental setup is shown in figure 6 . It contains two position controlled industrial robots manutec 33 with 6 degrees of freedom, equipped with force/torque sensors from Lord corp. with a measurement range of $660 \mathrm{~N}$ and $60 \mathrm{Nm}$ 


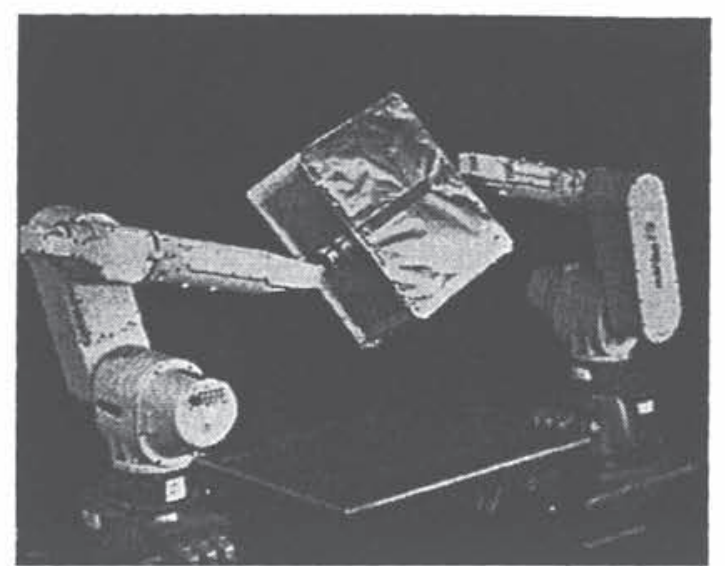

Figure 6. Experimental setup

and an accuracy of $0.5 \mathrm{~N}$ and $0.03 \mathrm{Nm}$. The cartesian sampling times $(10 \mathrm{~ms})$ of both robots have been synchronized. The delay time for the whole control cycle, including the sensors, robots, and coordination controller, amounts to 4 sampling steps, i.e. $40 \mathrm{~ms}$.

The task the system has to perform is to grasp an object - here a rectangular box is used which can be interpreted for example as orbital replacement unit - behind the table and to put it on the table by carrying out a transfer motion (see figure 7). In detail the following sequence has to be realized: The object has to be localized, which is done by a vision system mounted at the ceiling, then it has to be grasped firmly for achieving a force closure grasp - both is not within the scope of this paper -. Afterwards the compliance matrix to be used in the coordination controller has to be identified and the transfer motion has to be carried out. Figure 7 shows three views of the task execution, namely the approaching of the robots, while the object is in its initial position (a), a view during the transfer motion (b) and the object being released in the final position (c).

Here we will concentrate on the identification of the introduced transformed compliance matrix and we will show, that by use of this matrix the control performance during the transfer motion is improved considerably. Due to the assumption of unknown compliance of the system the active compliance controller contains an underestimated compliance matrix ${ }^{5} \boldsymbol{S}^{+}$, which leads to small gain coefficients and insures the stability of the system. The scalar gain factor $\frac{k_{i n t}}{T_{i n t}}$ has been choosen by pole placement for a well adjusted compliance matrix ${ }^{6}$. As the motion has been carried out without external contact, the external controller has been left out which in total led to the following compliance controller:

$$
\Delta x_{\mathcal{H}}=\frac{k_{\text {int }}}{T_{\text {int }} \cdot s} \boldsymbol{S}^{+} \boldsymbol{P}_{\text {int }}
$$

At the beginning of the transfer motion the compliance matrix i.e. the pseudoinverse $S^{+}$has to be identified, which is done by excerting small internal motions with the robots $\left(\delta \boldsymbol{x}_{\mathcal{H} S} \in \mathrm{R}_{i}\right)$ after having lifted the object for

\footnotetext{
${ }^{5}$ Here the pseudoinverse of the stiffness matrix as special form of the generalized inverse in equation (12) and (20) is used.

6 Due to measuring errors in the experimental setup only the damper term of (12) has been used.
}

some millimeters. For this contact case of the object, the free motion, the space of all admissible forces is reduced to the internal space ( $\boldsymbol{B}_{\text {eif }}=\boldsymbol{B}_{i}$ for $m=0$ ). Therefore an internal motion is sufficient for the persistent excitation, which is necessary for a good convergence of all parameter estimation algorithms - here a discrete square root filtering algorithm in information form because of it's excellent numerical properties is used The transformed matrix $S_{i}^{-1}$ is identified by transforming the measured arm motions and forces to the space of admissible forces according to (16) and (17), which for this contact case are changed to

$\delta \boldsymbol{f}_{i}=\boldsymbol{B}_{i}^{\mathrm{T}} \delta \boldsymbol{f}_{\mathcal{H}} ; \delta \boldsymbol{x}_{i}=\boldsymbol{B}_{i}^{\mathrm{T}} \delta \boldsymbol{x}_{\mathcal{H} 0}$, and $\delta \boldsymbol{x}_{i}=\boldsymbol{S}_{i}^{-1} \delta \boldsymbol{f}_{i}$.

After about 500 sampling steps i.e. $5 s$ the algorithm had estimated the parameters of $S_{i}^{-1}$ with an average error of about 3 percents of the biggest eigenvalue of the matrix. By use of the identified matrix the pseudoinverse is calculated according to (19) while taking into account, that for this contact case the formula is changed to $S^{+}=$ $\boldsymbol{B}_{i} \boldsymbol{S}_{i}^{-1} \boldsymbol{B}_{i}^{\mathrm{T}}$, and is incorporated into the controller.

Figure 9 demonstrates the improvement of the control performance when using the identified matrix instead of the initial, underestimated one. The object has to be moved along the trajectory shown in the Figures 7 and 8 . In detail first the object has to be lifted by $0.6 \mathrm{~m}$ (chang-

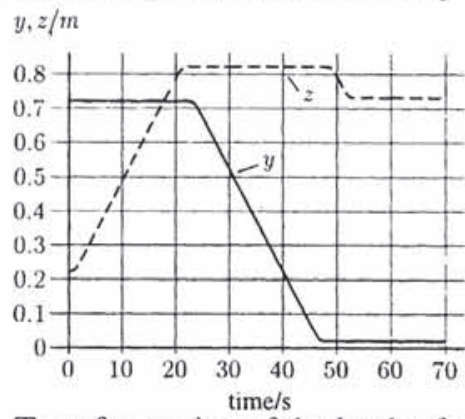

Figure 8. Transfer motion of the load reference point

ing z-component) then it has to be moved over the table (changing y-component) and finally it has to be placed on the table (changing $z$-component $)^{7}$.

Due to modeling errors ${ }^{8}$ the motion leads to undesired internal forces. Figure 9 a) and c) show the components of the internal force and torque error when using the initial, underestimated matrix while the pictures b) and d) show the force and torque errors respectively when using the identified matrix. As can be seen, the errors could be decreased considerably.

The most important component is the translational force in $x$-direction, being the axis connecting the two grasping points. In this direction the stiffness parameters of the system are very high, which means, that position errors lead to a high force error. With the initial matrix a squeezing force error in $x$-direction occurs up to $-65 \mathrm{~N}$,

\footnotetext{
7 In this example for sake of clear arrangement only a translational motion has been commanded, but of course also rotational motions can be applied, e.g. figure 6 displays a view from a combined translational and rotational motion.

8 At the used speed of $3 \mathrm{~cm} / \mathrm{s}$ mainly kinematic errors occur.
} 
a)

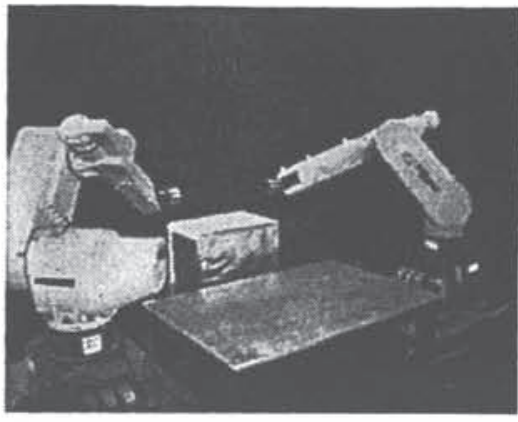

b)

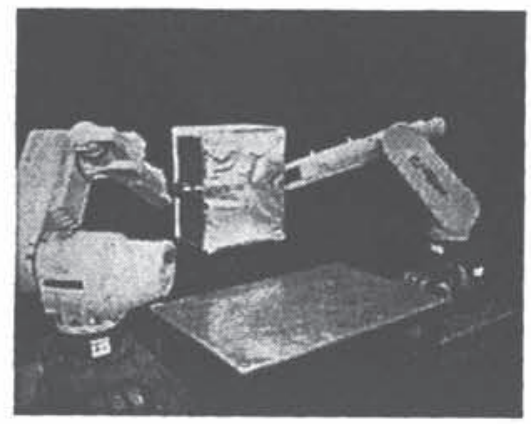

c)

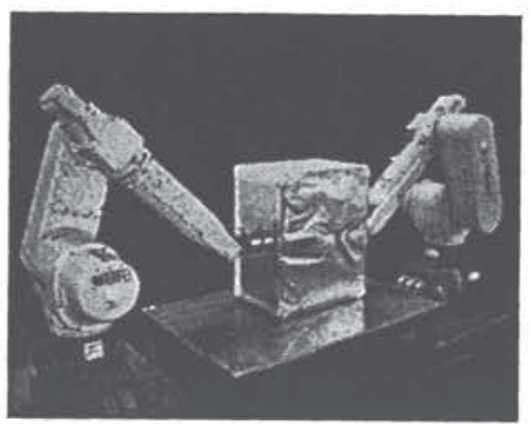

Figure 7. Grasping and transportation of an object.

a)

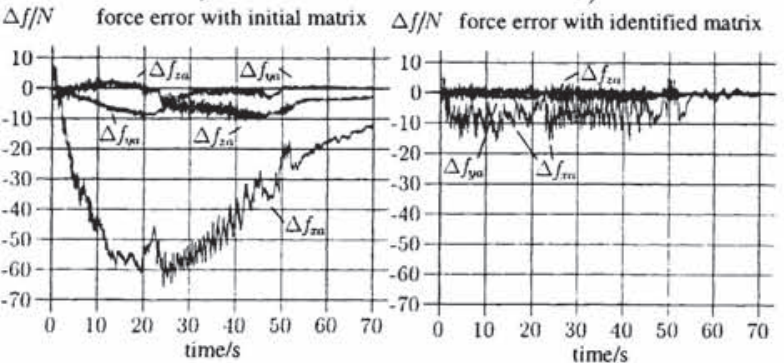

c)

$\Delta m / N m$ torque error with initial matrix $\Delta m / N m$ torque error with identified matrix

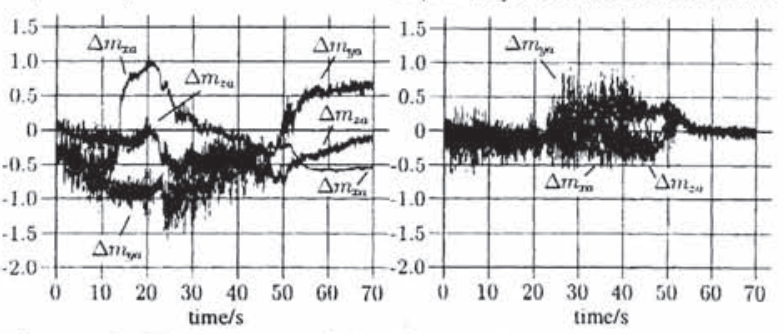

Figure 9. Trajectory of the internal translational force error (a) and (b) and torque error (c) and (d) occuring at a transfer motion, when using the initial, underestimated matrix (a) and (c) and the identified matrix (b) and (d)

at an offset force of $-100 \mathrm{~N}$, which is needed for ensuring a stable grasp of the object. Whereas the transfer motion in this direction can also be conducted with the initial matrix - only the control performance is increased by using the identified compliance matrix, and in some cases the object might be damaged due to the high internal force -, the inverse motion from the table to the starting position behind the table decreases the offset force by about the same error of $65 \mathrm{~N}$, leading to an entire force of about $-35 \mathrm{~N}$, where the object slips and is dropped.

\section{CONCLUSIONS}

A selftuning active compliance controller for a multi arm robot system handling a concerning it's compliant behaviour partly unknown object has been presented. The mathematical model of the system under consideration has been derived for different cases of the external contact of the object and a scheme has been formulated capable of determining the unknown compliance of the system, which is used in the controller. It has been shown by experiments for the case of two robots handling an unconstrained object, that by use of the identified compliance matrix the control performance of the two arm system could be improved considerably. The system is now capable of handling concerning its parameters only partly known objects, which is a step towards systems needed in non-industrial areas like space.

\section{REFERENCES}

von Albrichsfeld, C. (1996). Self-adjusting active compliance controller for two cooperating robots handling a flexible object. In Proceedings of the World Automation Congress (WAC'96). Robotics and Manufacturing Systems, France.

von Albrichsfeld, C. (1998). Ein Beitrag zur selbsteinstellenden Nachgiebigkeitsregelung für kraftschlüssig kooperierende Roboter. VDI-Verlag GmbH, series 8, No. 691, Germany.

von Albrichsfeld, C., Svinin, M., and Tolle, H. (1995). Learning approach to the active compliance control of multi-arm robots coupled through a flexible object. In Proceedings of the 3rd European Control Conference ECC'95, Italy.

Bruhm, H. and Neusser, K. (1987). An active compliance scheme for robots with cooperating arms. In Third International Conference on Advanced Robotics, Versailles, France.

Doty, K. L., Melchiorri, C., and Bonivento, C. (1993). A theory of generalized inverses applied to robotics. The International Journal of Robotics Research, 12(1):1 - 19.

Hayati, S. (1986). Hybrid position/force control of multi-arm cooperating robots. In Proceedings of the IEEE International Conference on Robotics and Automation, San Francisco, USA

Pandian, S. R., Kawamura, S., and Nakamura, K. (1994). Force/position learning control of multiple robot manipulators. In Proceedings of the Japan-USA Symposium on Flexible Automation, pages 955-962, Kobe, Japan.

Perdereau, V., Dauchez, P., and Douin, M. (1996). Experimental comparison of hybrid position/force control schemes for two cooperative robotic arms. In Symposium on Robotics and Cybernetics, pages $471-475$, Lille, France.

Svinin, M. and von Albrichsfeld, C. (1995). Analysis of constrained elastic manipulations. In Proceedings of the IEEE/RSJ International Conference on Intelligent Robots and Systems, volume 2 , pages 414-421, Pittsburgh, USA.

Svinin, M. M. and Uchiyama, M. (1994). Cartesian-level control strategy for a system of manipulators coupled through a flexible object. In Proceedings of the IEEE/RSJ International Conference on Intelligent Robots and Systems, volume 1, pages 687-694. München, Germany.

Szewczyk, J., Morel, G., and Bidaud, P. (1996). Optimization of cooperating system performances under distributed impedance control. In Symposium on Robotics and Cybernetics, pages 476481, Lille, France.

T.J. Tarn, A.K. Bejczy, and X. Yun (1986), "Coordinated Control of Two Robot Arms," in Proc. IEEE Int. Conf. on Robotics and Automation, USA

Uchiyama, M. and Dauchez, P. (1988). A symmetric position/force control scheme for the coordination of two robots. In Proceedings of the IEEE International Conference on Robotics and Automation, pages 350-356, Philadelphia, Pennsylvania, USA. 